\title{
TU/e EmHOONEN

\section{Step-wise excitation for the determination of the resonance frequency of a microparticle confined in a low pressure plasma}

\section{Citation for published version (APA):}

Meijaard, P., Staps, T. J. A., \& Beckers, J. (2021). Step-wise excitation for the determination of the resonance frequency of a microparticle confined in a low pressure plasma. Physics of Plasmas, 28(8), [083502]. https://doi.org/10.1063/5.0057509

DOI:

$10.1063 / 5.0057509$

Document status and date:

Published: 02/08/2021

\section{Document Version:}

Publisher's PDF, also known as Version of Record (includes final page, issue and volume numbers)

\section{Please check the document version of this publication:}

- A submitted manuscript is the version of the article upon submission and before peer-review. There can be important differences between the submitted version and the official published version of record. People interested in the research are advised to contact the author for the final version of the publication, or visit the $\mathrm{DOI}$ to the publisher's website.

- The final author version and the galley proof are versions of the publication after peer review.

- The final published version features the final layout of the paper including the volume, issue and page numbers.

Link to publication

\section{General rights}

Copyright and moral rights for the publications made accessible in the public portal are retained by the authors and/or other copyright owners and it is a condition of accessing publications that users recognise and abide by the legal requirements associated with these rights.

- Users may download and print one copy of any publication from the public portal for the purpose of private study or research.

- You may not further distribute the material or use it for any profit-making activity or commercial gain

- You may freely distribute the URL identifying the publication in the public portal.

If the publication is distributed under the terms of Article 25fa of the Dutch Copyright Act, indicated by the "Taverne" license above, please follow below link for the End User Agreement:

www.tue.nl/taverne

Take down policy

If you believe that this document breaches copyright please contact us at:

openaccess@tue.nl

providing details and we will investigate your claim. 


\title{
Step-wise excitation for the determination of the resonance frequency of a microparticle confined in a low pressure plasma
}

Cite as: Phys. Plasmas 28, 083502 (2021); doi: 10.1063/5.0057509

Submitted: 27 May 2021 - Accepted: 12 July 2021 .

Published Online: 2 August 2021 · Corrected: 3 August 2021

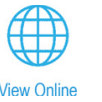

P. Meijaard, ${ }^{\text {a) }}$ (D) T. J. A. Staps, (D) and J. Beckers (D)

\author{
AFFILIATIONS \\ Department of Applied Physics, Eindhoven University of Technology, PO Box 513, Den Dolech, 5600 MB Eindhoven, \\ The Netherlands
}

${ }^{a)}$ Author to whom correspondence should be addressed: P.Meijaard@tue.nl

\begin{abstract}
We propose, test, and analyze a new step-wise excitation approach to quickly obtain, or determine with high accuracy, the resonance frequency of a microparticle confined in the space charge region of a low pressure plasma operated above a horizontal surface. The local value of this resonance frequency can directly be related to the charge of such a plasma-immersed microparticle and is, therefore, essential to be obtained with respect to the interpretation of many dusty and complex plasma laboratory experiments. The presented approach applies a step-wise change in plasma power, after which the damped harmonic oscillatory motion of the plasma-confined microparticle toward its "new" equilibrium confinement position is monitored temporally resolved and fitted with the theory describing this damped harmonic oscillator. Proof-of-principle experiments demonstrate that the results from this method match the results of the traditional frequency sweep resonance method within 5\%. Comparing to existing-for instance, phase-resolved or single particle injection-methods to determine the resonance frequency, this approach offers the experimenter a unique possibility to choose the trade-off between reduced measurement time-essential when working with particles that are vulnerable to plasma etching-and measurement accuracy.
\end{abstract}

Published under an exclusive license by AIP Publishing. https://doi.org/10.1063/5.0057509

\section{INTRODUCTION}

The physics of complex and dusty plasmas-i.e., the study of small (nanometer- to micrometer-sized) solid and charged particles confined in low pressure plasmas-has been an active research topic for several decades already. ${ }^{1-3}$ The related ecosystems studied can roughly be divided in two subsets.

The first subset includes systems in which particles are grown inside reactive gas-plasmas, such as silane, ${ }^{4}$ methane, ${ }^{5}$ acetylene, ${ }^{6,7}$ and hexamethyldisiloxane. ${ }^{8}$ Once grown sufficiently large, these particles may be confined in the positive plasma potential due to the permanent negative charge they obtain from the plasma bulk. In these so-called dusty plasmas, particle sizes range from the size of large molecules up to several micrometers while particle number densities as high as $10^{15} \mathrm{~m}^{-3}$ are found depending on the plasma parameters and the phase in the particle formation process. ${ }^{9}$ The reactive plasmas, in which these particles can be grown, have been recognized to have numerous applications ranging from the synthesis of nanostructures ${ }^{10}$ to thin film deposition. ${ }^{11}$

The second subset of ecosystems-often referred to as complex plasmas-includes plasmas, which contain a countable number of particles, often externally injected. The used particles in such systems are often well-defined in terms of morphology, size, and material composition. The advantage of such systems is that the cloud of confined microparticles in this regime may lead to strongly coupled systems, ${ }^{12}$ allowing them to be used as macroscopic systems to study fundamental phenomena, such as crystallization, ${ }^{13}$ phase transitions, ${ }^{14}$ and (dust) density waves under both laboratory conditions on Earth, ${ }^{15}$ and under microgravity conditions in the International Space Station. ${ }^{16}$

The fundamental importance of micrometer-sized particles inserted in plasmas is based on the fact that they become electrically charged by the collection of plasma species and may levitate in the space charge region-often called the plasma sheath-which is selfinduced by the plasma upon contact with a surface. Surfaces immersed in a plasma are usually charged negatively due to the higher mobility of electrons compared to that of the ions. This effect creates a region where electrons are repelled in the vicinity of the surface. Hence, a thin layer, with a thickness of typically 1 to $10 \mathrm{~mm}$ for low temperature and low pressure plasmas, is created in which the combination of depleted electrons and (streaming) ions leads to the buildup of a space charge, 
which, in turn, leads to high, local, and time-averaged electric fields and a positive plasma potential compared to the plasma-facing surface potential.

Despite the importance of the presence of the plasma sheath for many industrial applications, such as those in the solar cell industry, ${ }^{17}$ lithography, ${ }^{18}$ material processing, ${ }^{19}$ glass fiber production, ${ }^{20}$ contamination control, ${ }^{21}$ the exploration of this region has dramatically appeared complex. Traditional probe techniques have appeared too invasive $^{22}$ and optical-Stark-based-techniques ${ }^{23,24}$ suffer from insufficient signals due to too low electric fields present in the sheath region.

To experimentally explore the sheath region of such low pressure plasmas, the aforementioned microparticles have been utilized as (almost) noninvasive probes in several studies. ${ }^{25-30}$ From a basic point of view, a microparticle will-after being injected into and charged by the plasma-levitate at the exact position in the sheath region where the sum of all (plasma-induced) forces working on it is zero. With information about the microparticle charge, one could learn more about the relevant fundamental plasma-particle interactions and the different (plasma-induced) forces working on the microparticle.

As becomes clear from the above, for both the analysis and interpretation of complex plasmas (and connected research efforts in general), and the interpretation of the plasma sheath probing experiments using microparticles, the particle charge is a crucial parameter to determine. However, since the particle charge is mutually intertwined with most of the fundamental plasma-particle interactions, determining that this charge is a complex task. Traditional theoretical frameworks to determine this charge-for instance, based on the Orbital Motion Limited (OML) theory ${ }^{22}$-become less applicable in the sheath region because in this, quasi-neutrality does not hold and high electric fields, inherently present there, induce an ion flow. All these effects complicate the modeling of the particle charge and demand for experimental data to reveal new insights and to obtain additional information.

Up to now, several experimental approaches have successfully been developed and applied to this challenge. For instance, tracking the temporal evolution of the interaction between two Coulombcolliding particles can give insight about the charge that these particles carry with them. ${ }^{31}$ Optical tweezers, i.e., complex setups where two counter propagating laser beams trap a single particle by laser pressure in the plasma sheath or bulk, or even without plasma present, have been developed by Schneider and Kersten. ${ }^{32}$ Tracking the damped oscillatory trajectories of microparticles toward their equilibrium confinement position upon external injection in the plasma was studied by Tomme et al. $^{33}$ From such trajectories, those authors obtained information regarding the local value of the microparticle's charge. Another trajectory based technique used an ion source to alter the vertical trajectories of injected hollow microspheres to investigate the influence of fast neutrals in the ion beam on the microparticles. ${ }^{34}$ Maybe the mostly used and most elegant way of determining the charge of plasma-confined microparticles is the resonance method, which was originally introduced by Melzer et al. ${ }^{35}$ and later modified by Homann et al. to investigate only one single microparticle in a crystal lattice. ${ }^{36}$ In the resonance method, a single plasma-confined particle is excited-for instance, by amplitude-modulating the driving radio frequency (RF) voltage-after which the response of the microparticle's motion to a frequency sweep (typically between 1 and $100 \mathrm{~Hz}$ ) reveals the "eigen-frequency" or undamped resonance frequency. This resonance frequency can then be related to the particle's charge once local plasma parameters are known, for instance, from modeling efforts. Zafiu et al. demonstrated that non-linear effects become significant for larger particle excitation amplitudes. ${ }^{37}$ Furthermore, a phase resolved resonance method for accurate fitting of both the real and imaginary parts of the resonance curve was introduced by Carstensen et al. ${ }^{38}$

Despite being useful and elegant, these resonance-based methods require a full frequency sweep with a sufficient spectral resolution in the spectral domain around the (expected) resonance frequency. From an experimental point of view, this takes considerable time to conduct, of course, depending on the desired resolution and measurement accuracy. Taking into account that most experiments in the literature use Melamine-Formaldehyde (MF) microparticles, which, in general, are considerably affected by plasma-etching, ${ }^{29,39,40}$ the total measurement time should be as short as possible to prevent the decreasing accuracy due to a drifting change in mass, particle size and, therefore, a drift in the exact position where the particle probes the plasma sheath.

Although Tomme et al. ${ }^{33}$ experimentally derived the resonance frequency for a single response in the time-domain, repeating the experiments with their particle drop technique to achieve higher accuracy would introduce additional uncertainties with respect to the measurement location, size (i.e., charge), and mass of the used microparticles. These uncertainties are introduced because each additional measurement requires a new microparticle to be injected for which the size and mass may differ with respect to the same properties of the particle used for the first experiment. Due to the spread in mass, not only a measurement error is introduced when it comes to the resonance frequency but also the exact location at which such microparticles fulfill their probing "duty."

In this work, we introduce and propose step-wise excitation of the probing microparticle after which the particle's response is monitored in the time-domain (rather than phase resolved). Analyzing this response provides information about the resonance frequency and damping, which, in turn, could be used to obtain information about the microparticle's charge and for the modeling and interpretation of various dusty and complex plasma experiments. This method of particle excitation can be applied to an already confined microparticle and is initiated by instantaneously changing the plasma power. In contrast to phase-resolved methods, only one single step excitation experiment could provide the needed information about the resonance frequency and damping. Hence, the measurement time would significantly be shortened while the experiment can be repeated for statistical accuracy depending on the desired measurement accuracy.

By applying the approach discussed in this work, the experimenter is given the freedom to choose the optimal trade-off between the total duration of the experiment and the increased accuracy achieved by repeating the experiment with exactly the same particle over and over again.

This work is organized as follows. Section II introduces some theoretical considerations regarding the plasma particle confinement and the response of a plasma-confined microparticle against a step excitation. The experimental approach-including the experimental hardware, the measurement procedure, and the data processing-is subsequently discussed in Sec. III. In Sec. IV, the primary results are shown after which a comparison is made with results obtained with the traditional resonance method (Sec. V). Section VI concludes this work. 


\section{THEORY}

As explained in Sec. I, the proposed method is based on the response of a single microparticle confined in the sheath region of a low pressure plasma after being excited by a step-wise change in the plasma power.

In this section, we first briefly summarize the mechanism behind microparticle charging in low pressure plasmas (Sec. II A) and the dominantly involved (plasma-induced) forces working on a microparticle leading to a stable equilibrium position in the plasma sheath (Sec. II B). A qualitative summary of the relevant forces is briefly given in Sec. II C. How the (local) particle resonance frequency is determined from the particle's response to the applied step-wise excitation is discussed from a theoretical perspective in Sec. II D.

\section{A. Charging of plasma-immersed microparticles}

Upon injection into a low pressure radio frequency (RF) driven discharge, a microparticle usually acquires a permanent negative surface charge $Q_{p}$ by collecting incoming plasma fluxes. Because the plasma is generated by an oscillating radio frequency (RF) electric field of $13.56 \mathrm{MHz}$, the electrons usually have a temperature of a few electron volts ${ }^{19}$ (i.e., a multiple of $11.6 \times 10^{3} \mathrm{~K}$ ) while the ions remain at room temperature. Initially, the highly mobile electrons collide with the injected particle more often than the ions do. As electrons stick to the surface of the microparticle, this process negatively charges the particle surface. The developing negative surface potential of the microparticle enhances the ion flux and suppresses additional electron fluxes toward the particle until a surface equilibrium potential (and related surface equilibrium charge) is reached at which the electron and ion fluxes equalize. This theoretical framework for determining the charge of a plasma-embedded microparticle-based on equalizing the electron and ion fluxes toward its surface-is called the Orbital Motion Limited (OML) theory ${ }^{22}$ and is applied throughout the field. In the quasi-neutral plasma bulk, plasma-embedded microparticles are predicted to obtain negative charges up to $10^{4}$ to $10^{5}$ elementary charges depending on parameters, such as the particle size and the temperature of the electrons. ${ }^{41}$ In most dusty and complex plasma experiments on Earth, gravity pulls the microparticle toward the lower boundaries of the plasma, i.e., in the case of this work toward the plasma sheath region just above the lower (horizontally aligned) electrode (see Fig. 1). In this region, the microparticle is embedded in a plasma region with a (time-averaged) depleted electron population. Encumbered by their inertia, the relatively heavy ions cannot follow the applied frequency of the electric field and, thus, are only subjected to the time-averaged sheath electric field induced by the plasma itself. This time-averaged electric field accelerates the ions toward the plasma-facing electrode. Looking back from the microparticle's point of view, these two phenomena-the depletion of the electron population and the sheath-electric-fieldinduced streaming of the ions-lead to the values of the microparticle equilibrium charge, which are up to two orders of magnitude less negative, compared to particle charges obtained in the quasineutral plasma bulk region. ${ }^{42}$ Additionally, even in weakly ionized plasmas as used here, ion-neutral collisions with the particle surface can alter the final value of the particle charge, making it less negative. ${ }^{43}$

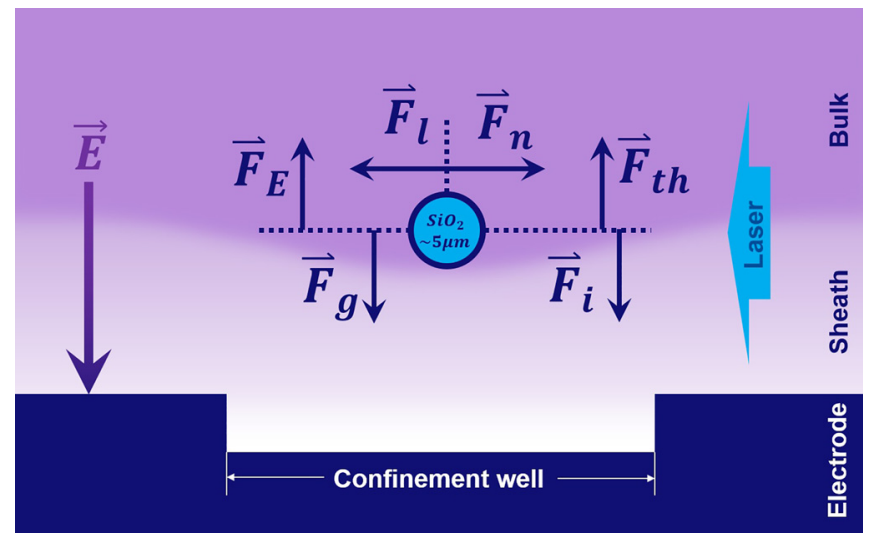

FIG. 1. Overview of force vectors and the driving phenomena, working on the particle probe confined in the plasma sheath.

\section{B. Dominant forces acting on plasma-immersed microparticles}

In this section, we briefly discuss the dominant forces acting on a single microparticle that is confined in the sheath region of a low pressure plasma. The ecosystem of a charged microparticle confined above a horizontally oriented (with respect to gravity) electrode in the plasma sheath region (as is the case in this work), and the forces working on the particle are schematically shown in Fig. 1.

\section{Electrostatic force}

As discussed, high and time-averaged electric fields $\vec{E}$ (up to $10^{4}$ to $\left.10^{5} \mathrm{~V} \mathrm{~m}^{-1}\right)^{42}$ are present in the plasma sheath region, which points from the plasma in the direction of the plasma-facing surface. This electric field acts on the negative particle charge $Q_{p}$, which results in an electrostatic force

$$
\vec{F}_{\mathrm{E}}=Q_{\mathrm{p}} \cdot \vec{E},
$$

which is directed away from the surface (see Fig. 1). Since both $Q_{\mathrm{p}}$ and $\vec{E}$ are functions of the particle confinement position, the electrostatic force $\vec{F}_{\mathrm{E}}$ is also position dependent.

\section{Ion drag force}

The sheath electric field $\vec{E}$ leads to a streaming flux of positive ions from the plasma toward the plasma-facing surface, which can exert momentum-and, thus, a force which is often called the ion drag force-to the microparticle either by direct impact or through Coulomb interactions.

The direct impact, or the collisional part, of the ion drag force, can be expressed as

$$
\vec{F}_{\mathrm{i}}^{\text {Coll }}=n_{\mathrm{i}} m_{\mathrm{i}} u_{\mathrm{i}} \vec{u}_{\mathrm{i}} \pi r_{\mathrm{p}}^{2}\left[1-\frac{2 e \phi\left(r_{\mathrm{p}}\right)}{m_{\mathrm{i}} u_{\mathrm{i}}^{2}}\right],
$$

where $n_{\mathrm{i}}$ is the local ion density, $m_{\mathrm{i}}$ is the ion mass, $\vec{u}_{\mathrm{i}}$ is the local ion velocity, $u_{\mathrm{i}}$ is its magnitude, $e$ is the elementary charge, $r_{\mathrm{p}}$ is the microparticle's radius, and $\phi$ is its equilibrium potential at the confinement position. ${ }^{41}$ 
The Coulomb scatter part of the ion drag force is due to the streaming ions, which closely pass by the charged microparticle and Coulomb-interact with it, but do not hit the microparticle's surface. As theoretically each passing ion is interacting with the microparticle, the cylindrically symmetric ion flow will exert a net ion drag force on the microparticle, which is generally pointing from the plasma toward the electrode surface. This part of the ion drag force is commonly expressed as ${ }^{44}$

$$
\vec{F}_{\mathrm{i}}^{\text {Coul }}=4 m_{\mathrm{i}} v_{\mathrm{i}, \text { tot }} n_{\mathrm{i}} \vec{u}_{\mathrm{i}} \pi b_{\pi / 2}^{2} \ln \left(\frac{\lambda_{\mathrm{s}}+b_{\pi / 2}^{2}}{r_{\mathrm{p}}+b_{\pi / 2}^{2}}\right),
$$

where $v_{\mathrm{i}, \text { tot }}$ is the total ion speed, which is the vector sum of the ion speed plus the thermal speed, ${ }^{45}$ and $\lambda_{\mathrm{s}}$ is the linearized Debye length given by $\lambda_{\mathrm{s}}=\frac{\lambda_{\mathrm{D}, \mathrm{e}} \cdot \lambda_{\mathrm{D}, \mathrm{i}}}{\sqrt{\lambda_{\mathrm{D}, \mathrm{e}}^{2}+\lambda_{\mathrm{D}, \mathrm{i}}^{2}}}$, in which $b_{\pi / 2}$ is the asymptotic impact parameter of which the asymptotic orbit angle is $\pi / 2^{46}$ and $\lambda_{\mathrm{D}, \mathrm{e} / \mathrm{i}}=\sqrt{\frac{\epsilon_{0} T_{\mathrm{e}, \mathrm{i}}}{e n_{\mathrm{e}, \mathrm{i}}}}$ is the electron/ion Debye length, respectively, where $T_{\mathrm{e}, \mathrm{i}}$ is the electron and ion temperature, $n_{\mathrm{e}}$ is the electron density, and $\epsilon_{0}$ is the permittivity of vacuum. The resulting vector sum of both subforces is referred to as the total ion drag force

$$
\vec{F}_{\mathrm{i}}=\vec{F}_{\mathrm{i}}^{\text {Coll }}+\vec{F}_{\mathrm{i}}^{\text {Coul }},
$$

which depends-as well-on the confinement position of the microparticle.

\section{Gravitational force}

The gravitational force only depends on the microparticle's mass and the Earth's gravitational field $\vec{g}$ and can be expressed by

$$
\vec{F}_{\mathrm{g}}=4 / 3 \pi r_{\mathrm{p}}^{3} \rho \vec{g},
$$

where $\rho$ is the microparticle's mass density. In the case of the currently presented experiments with a microparticle levitated above a horizontally aligned electrode (see Fig. 1), the gravitational force vector is pointing toward the electrode, away from the plasma.

\section{Other non-dominant forces}

Next to the above-mentioned dominant forces, there are several other forces known that may work on plasma-confined particles. These are-in the current experiment-non-dominant and include the thermophoretic force induced by thermal gradients in the system, the neutral drag force caused by momentum transfer from a neutral gas flow, and the radiation pressure force caused by the irradiating laser. Since no significant thermal gradients are present, gas flows are minimal near the particle's position and the used laser power is also low, these forces can be considered negligible with respect to the above-mentioned electric force, ion drag force, and gravitational force.

\section{Resulting microparticle confinement}

A qualitative overview of all previously mentioned forces acting on the probing microparticle is schematically shown in Fig. 1. This schematic view is made of the focus plane of the used high-speed camera as will be discussed in Sec. II D.
Under certain conditions, an injected microparticle may find a stable vertical confinement position at which the sum of all previously mentioned forces-for the case of this work usually working in the vertical direction-is exactly zero. It is well known from the literature that in plasma sheath regions, microparticles can be confined for hours and even longer. ${ }^{29}$

Although horizontal components of forces-such as the horizontal force exerted by the irradiation laser and small horizontal components of the gravitational force caused by a slight misalignment of the electrode with respect to the horizontal plane-are negligible with respect to the earlier mentioned dominant forces, they may result in loss of particles from the camera focus area in the horizontal direction. To prevent this, horizontal confinement is achieved by a confinement well, i.e., a $1.0 \mathrm{~mm}$ deep indent milled in the electrode's flat surface (elaborated on in Sec. III A). Since the space charge region slightly follows this indent, the particle is forced to be horizontally confined above the center of this indent.

\section{Particle's response after step excitation}

From an experimental point of view, in our proposed method, a single microparticle is first (e.g., for $t<0$ ) stably confined at its equilibrium position under steady-state plasma conditions. This means that under these initial plasma conditions, and at an equilibrium position of the microparticle, the sum of all plasma-induced forces working on the microparticle is zero.

At a certain moment in time (e.g., at $t=0$ ), the RF plasma driving voltage $V_{\mathrm{rf}}$ is instantaneously changed by a fraction $\Delta V_{\mathrm{rf}}$ and, with that, also the plasma power is suddenly changed. Driven by this step-wise change in the plasma power, the plasma sheath structure suddenly changes as well and, as a result, the position at which the microparticle would experience a zero net force-i.e., the micro particle's equilibrium position-is instantaneously redefined at another position.

Caused by the relatively large inertia of the microparticle compared to the electrons and ions in the plasma, the overall response of the plasma to this sudden change-which occurs at time scales on the order of $100 \mu$ s (Ref. 47) - is much faster than the response of the microparticle itself to the changing environment it is embedded in. Hence, this yields decoupling of the plasma and microparticle dynamics time scales. In contrast, in the aforementioned traditional phase-resolved particle resonance methods, the microparticle is excited by amplitude modulating the plasma with a frequency comparable to the eigen-frequency of the microparticle. Consequently, this may lead to coupling of the plasma and microparticle dynamics time scales.

Now, for $t \gg 100 \mu \mathrm{s}$, the microparticle starts to move toward its new equilibrium position in a plasma environment, which has been changed with respect to $t<0$, but can be considered steady-state. The microparticle will display a damped harmonic oscillating motion toward this new equilibrium position. By carefully analyzing the microparticle's trajectory and by comparing and fitting it with the theory, both the local resonance frequency and the damping ratio can be retrieved as explained below.

Assuming a linear restoring force, after excitation by the step in $\mathrm{RF}$ driving voltage and RF power, the following equation of motion applies to the confined microparticle: 


$$
m_{\mathrm{p}} \cdot \ddot{z}+2 \gamma m_{\mathrm{p}} \cdot \dot{z}+m_{\mathrm{p}} \omega_{0}^{2} \cdot z=\left\{\begin{array}{ll} 
\pm \alpha & t \leq 0 \\
0 & t>0
\end{array},\right.
$$

where $z(t)$ is being the time-dependent microparticle position above the electrode, $\dot{z}$ and $\ddot{z}$ are the first and second order derivatives of $z$ with respect to time $t$, respectively, $m_{\mathrm{p}}$ is the microparticle's mass, $\gamma=\zeta \cdot \omega_{0}$, which is the product between the damping ratio $\zeta$ and the natural/undamped angular resonance frequency $\omega_{0}$ of the probing microparticle. $\alpha$ is the perturbation of force (due to the difference in $\mathrm{RF}$ driving power) to have the microparticle confined at the pre-step equilibrium position. The solution of the equation of motion of the microparticle after the step-excitation toward its equilibrium position (which is to be probed) is given by ${ }^{48}$

$$
z(t)=D \cdot e^{-t / \tau} \cdot \cos \left(\omega_{\mathrm{d}} \cdot t+\phi_{s}\right)+z_{0}\left(10^{-3} \mathrm{~m}\right),
$$

in which $D$ is the arbitrary initial amplitude of the response, $\omega_{\mathrm{d}}$ is the damped angular resonance frequency, $\tau$ is the decay time constant of the oscillation amplitude, $z_{0}$ is the new or post-step probing microparticle's equilibrium position, and $\phi_{\mathrm{s}}$ is the starting phase at $t=0$, which is defined as

$$
\phi_{\mathrm{s}}=\left\{\begin{array}{llll}
\pi / 2 & \text { "falling" } & (\alpha>0) & (\mathrm{rad}) \\
3 \pi / 2 & \text { "rising" } & (\alpha<0) & (\mathrm{rad}) .
\end{array}\right.
$$

The damped angular resonance frequency is related to the natural angular frequency by ${ }^{33}$

$$
\omega_{\mathrm{d}}=\omega_{0} \sqrt{1-\zeta^{2}}\left(\mathrm{rad} \mathrm{s}^{-1}\right)
$$

The decay time constant $\tau$ in the exponent of Eq. (7) is related to the damping ratio by

$$
\tau=\frac{1}{2 \pi \zeta \cdot f_{0}}(s)
$$

where $f_{0}$ is the natural frequency of the microparticle. Rewriting the above relation for $\zeta$, substitution of $\omega_{\mathrm{d}, 0}=2 \pi \cdot f_{\mathrm{d}, 0}$ and some algebra, Eq. (9) can be solved for the natural resonance frequency $f_{0}$ as

$$
f_{0}=\sqrt{f_{\mathrm{d}}^{2}+\left(\frac{1}{2 \pi \tau}\right)^{2}}\left(\mathrm{~s}^{-1}\right) .
$$

From fitting Eq. (7) to the experimentally obtained data (i.e., the timeresolved particle trajectory after step excitation), the time decay constant $\tau$ and the damped resonance frequency $f_{\mathrm{d}}$ can be found, which, in turn, delivers the natural frequency $f_{0}$ by using Eq. (11). From this framework, no difference in the solution given by Eq. (7) is expected between situations for which $\alpha$ is positive and situations for which $\alpha$ is negative.

\section{EXPERIMENTAL}

All experiments presented in this work have been performed on one and the same single microparticle levitated in the sheath region of a parallel plate, capacitively coupled low pressure argon plasma.

In this section, the overall experimental setup will be described in Sec. III A, followed by a discussion of the procedure that was followed to obtain the proof-of-principle measurements in Sec. III B. Finally, the post-processing of the data from the high speed camera system is briefly discussed in Sec. III C.

\section{A. Experimental setup}

A schematic overview of the overall setup used for the experiments-including a vacuum vessel, particle injection, illumination and tracking equipment, and gas handling-is depicted in Fig. 2. Centered inside this vacuum vessel is the electrode configuration, which is depicted in greater detail in Fig. 3.

The used vacuum vessel shown in Fig. 2 had a volume of $\sim 4 \mathrm{~L}$ and contained three ISO K-100 and two ISO K-160 ports. In the vacuum vessel, the background pressure was $<1 \times 10^{-4} \mathrm{mbar}$, which was checked before the measurements using a Hornet IGM400. During the experiments, the operating argon gas pressure in which the plasma was created was $16.2 \pm 1.6 \mathrm{~Pa}$ as measured using a CVG101 Worker Bee [Fig. 2(b)] pressure gauge.

The inlet of gas was controlled by a Brooks GF40 mass flow controller with full scale flow capacity of 10 SCCM. This gas left the vessel through a stationary HiPace 80 turbo pump [Fig. 2(d)], which was directly connected to the vacuum vessel. Down the vacuum line, two vacuum valves in succession choked the exhaust gas flow toward an operating HiPace 80 . This turbo molecular pump was backed by a LeyBold Scrollvac 10.

The electrode assembly [Fig. 2(g)] was mounted on the bottom flange of the $18 \mathrm{~cm}$ cube vacuum vessel of black anodized aluminum as shown in Fig. 2.

The plasma discharge was generated between two, a $5.0 \mathrm{~mm}$ thick, $70 \times 70 \mathrm{~mm}^{2} 316 \mathrm{~L}$ stainless steel electrodes with rounded edges, which were aligned in the horizontal plane, and they were separated by a $40 \mathrm{~mm}$ distance from one another (see Fig. 3). The top electrode [Fig. 3(a)] was grounded via the wire frame [Fig. 3(f)], which held both electrodes in place. The $15 \mathrm{~mm}$ hole in the middle of this top electrode [Fig. 3(b)] granted from the top injected microparticles access to the discharge region, to have them finally reaching the sheath region of interest just above the bottom electrode. The bottom electrode

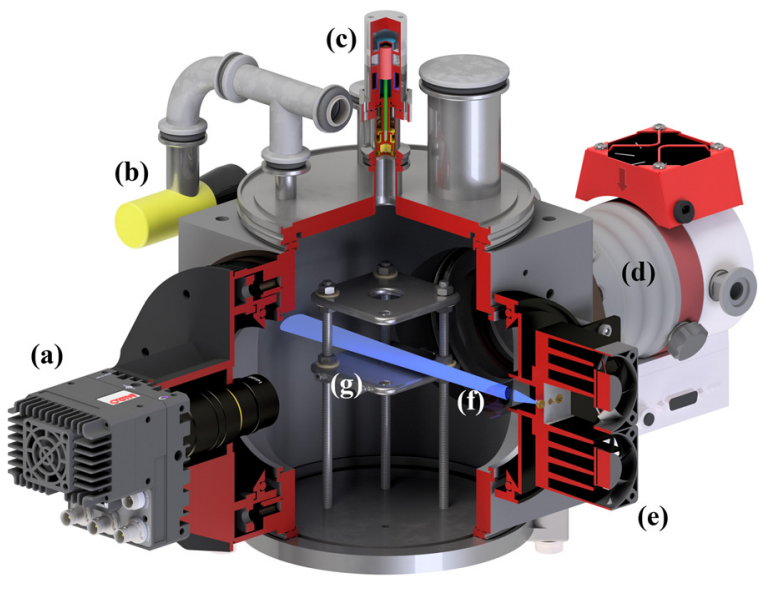

FIG. 2. Complete vacuum vessel, with the (for visual purposes) cut surfaces shown in red: (a) high speed camera and optics, (b) pressure gauge, (c) in-house developed particle injector, (d) stationary turbo molecular pump, (e) laser assembly, (f) laser beam, and $(\mathrm{g})$ electrode assembly. 


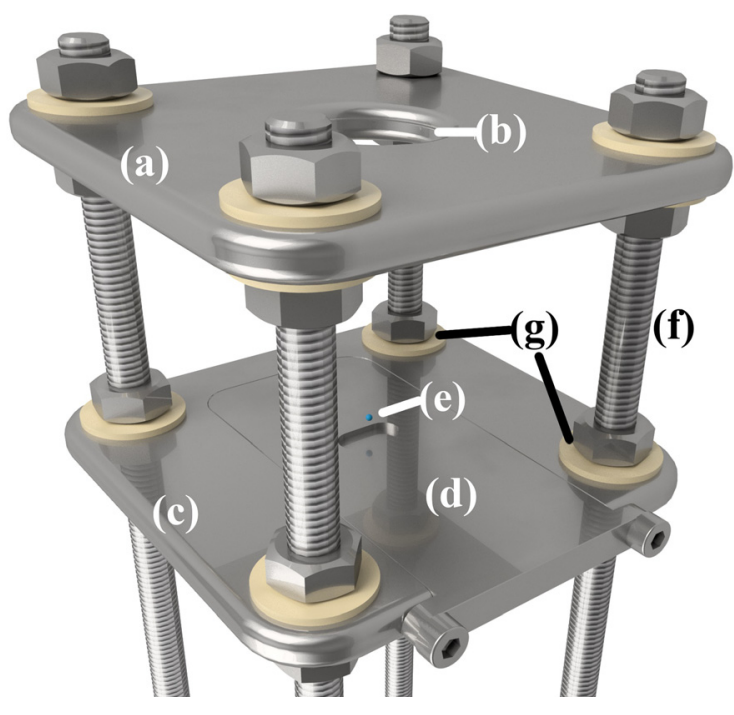

FIG. 3. Close-up of the RF electrodes, with: (a) grounded electrode, (b) the $15 \mathrm{~mm}$ hole, (c) powered electrode, (d) exchangeable inset, (e) particle probe, (f) wire frame, and (g) PEEK insulators.

[Fig. 3(c)] was powered with a radio frequency (RF) voltage signal and was electrically insulated from the wire frame by customized PEEK insulating parts [Fig. $3(\mathrm{~g})$ ]. A $1 \mathrm{~mm}$ deep, $5 \times 10 \mathrm{~mm}^{2}$ slot-shaped indent was milled in an exchangeable insert [Fig. 3(d)], which was fixed in the powered bottom electrode to create a potential well to ensure that the probing microparticle remained just above the center of the bottom electrode [Fig. 3(e)], i.e., in the limited depth of field of the optical system.

The plasma power was generated by using two function generators in succession: the first function generator's output of -5 to $5 \mathrm{~V}$ was fed to the modulation input of the second function generator. The second function generator provided a constant $13.56 \mathrm{MHz}$ carrier signal, which was amplitude-modulated by a set percentage by the output of the first function generator. This modulated RF signal was sent to a Mini Circuits LZY-22+ amplifier, which, via a MFJ Versa Tuner II (model: MFJ-969), applied the RF voltage on the bottom electrode of Fig. 3. The plasma power was determined by an Octiv Poly VI probe, which was installed in the RF line prior to the measurements.

The used microparticle was made from $\mathrm{SiO}_{2}$ (which are pristine argon plasma resistant ${ }^{39,49}$ ) and had a diameter of $4.96 \pm 0.20 \mu \mathrm{m}$, with a manufacturer (Microparticles $\mathrm{GmbH}$ ) reported mass density of $1850 \mathrm{~kg} \mathrm{~m}^{-3}$. The microparticle was injected in the same way as was done by Beckers et al. ${ }^{29,42,50,51}$ before. This was done by a "salt" shaker like device [Fig. 2(c)], which had two $\approx 40 \mu \mathrm{m}$ holes in its bottom plate. To activate the shaker, a brief pulse of $24 \mathrm{~V}$ was put on its atmospheric coil, which suddenly attracted the particle container inside the vacuum, to be returned to its initial position by a spring after the pulse.

The laser beam [Fig. 2(f)] used to illuminate the microparticle was generated by a $450 \mathrm{~nm}$ laser diode (Thorlabs L450P1600 MM), which was compressed in the direction of the perpendicular axis $\left(\theta_{\perp}\right)$ by a cylindrical lens with $f=24.88 \pm 0.25 \mathrm{~mm}$, with the laser diode situated at the focal line of the lens [Fig. 2(e)]. The CW laser beam power at the position of the particle was roughly $5 \mathrm{~kW} \mathrm{~m}^{-2}$. The laser beam was finally dumped on the anodized blind side of the vacuum vessel.

The camera used for imaging was a monochrome, 12-bit Phantom Miro C110 [Fig. 2(a)]. This camera was fitted with a bandpass filter (center wavelength of $450 \mathrm{~nm}$ and a FWHM of $10 \mathrm{~nm}$ ) and "machine vision" optics, Thorlabs MVL50TM23, with a fixed focal length of $f=50 \mathrm{~mm}$. The resulting spatial resolution at the position of the probing microparticle was $16.5 \mu \mathrm{m} /$ pixel.

\section{B. Measurement procedure}

Before the start of an experiment, the vacuum vessel as shown in Fig. 2 was pumped down to the base pressure and an argon gas flow of $\approx 0.6$ SCCM was introduced in the vessel while the pressure in the vessel was allowed to stabilize at an operating pressure of $16.2 \pm 1.6 \mathrm{~Pa}$. Then, the plasma was switched on using an RF power of $3.2 \pm 0.3 \mathrm{~W}$ after which the system was allowed to thermally stabilize for roughly $3 \mathrm{~h}$. After these $3 \mathrm{~h}$ of plasma-on time, several microparticles were injected into the plasma vessel by using the particle injector. By tilting the relatively small vacuum vessel, more and more microparticles were allowed to escape from the confining horizontal potential well above the powered electrode until a single particle remained trapped. This remaining single microparticle was the particle with which all experiments presented in this manuscript have been conducted.

The absolute value of the applied step in RF power was chosen such that the microparticle would not move too quickly for the camera to resolve it (raw data shown in Fig. 4), which was smaller than $15 \mathrm{~mm} \mathrm{~s}^{-1}$ in our most extreme case. However, the step in power was chosen sufficiently large to be able to resolve several periodic oscillations of the microparticle toward its new equilibrium position after excitation. This is more extensively discussed in Sec. VB.

The post-step plasma power used to achieve a suitable microparticle equilibrium position $z_{0}$ was $3.2 \pm 0.3 \mathrm{~W}$. For the measurements in which the plasma power was suddenly increased to this value-in this work these experiments are indicated as rising step excitation-the pre-step plasma power was set to $1.5 \pm 0.2 \mathrm{~W}$. The corresponding prestep equilibrium position of the microparticle is denoted by $z_{1}$. Similarly, for the measurements in which the plasma power was suddenly decreased to the value of $3.2 \pm 0.3 \mathrm{~W}$-in this work these experiments are indicated as falling step excitation-the pre-step plasma power was set to $6.6 \pm 0.7 \mathrm{~W}$. The corresponding pre-step equilibrium position of the microparticle is denoted by $z_{\mathrm{h}}$.

Statistical information has been obtained by using a modulation signal, which was a $2 \mathrm{~Hz}$ square wave with a duty cycle of $50 \%$. The repetition rate was sufficiently low to ensure fully restored and stable plasma confinement of the involved microparticle. The high speed camera was triggered on the rising/falling edge of the modulation voltage, until the memory section $(\approx 183$ s) of the camera was saturated. The set high speed camera settings were as follows: 180 Frames Per Second (FPS), with an exposure of $5.55 \mathrm{~ms}$ per frame at the maximum exposure index of 25000 .

\section{Data processing}

The amount of data is drastically reduced by taking two pictures of the stably levitating microparticle probe, with different camera frame sizes. In the first image, the microparticle and the electrode were 

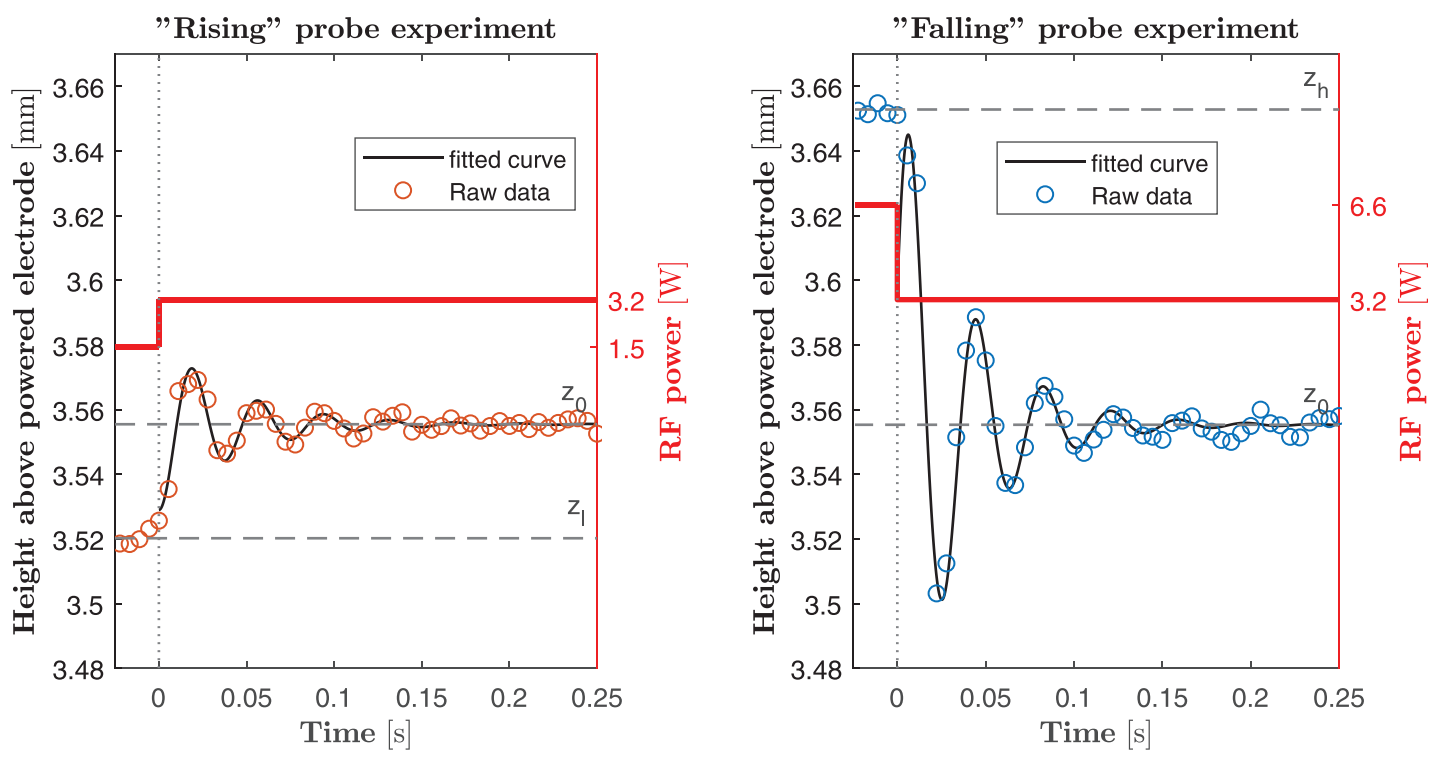

FIG. 4. The $\mathrm{SiO}_{2}$ microparticle's response when excited from an instantaneous lower (left) and higher (right) plasma power, together with fits of Eq. (7) and the equilibrium positions before $\left(z_{l}\right.$ and $z_{h}$ for rising and falling step excitation) and after $\left(z_{0}\right)$ the step excitation. The applied RF power is depicted in red.

photographed in one frame. The second image was taken with a smaller frame size, which only included the microparticle. Because the microparticle probe was verified to be at a stationary location, the two pictures were related to one another and, from that, the height of the exact position of the microparticle could be determined without having to record the electrode in each frame. By this referencing between the overall and small photographs, almost a 30 -fold data reduction per frame was achieved. The additional error in the particle position made by this data-saving approach is assumed to be less than 1 pixel or $16.5 \mu \mathrm{m}$. The nature of this error is a systematic one and is, therefore, not shown in the results in this work.

The referenced images were processed by the MATLAB software developed by van Minderhout et al., ${ }^{21}$ after being adapted to the tracking of a single particle. For each consecutive video frame, this software enabled to resolve, with sub-pixel resolution, the particle position despite the resolution of the optical system used. The resulting particle locations were fitted with Eq. (7) in the time-domain by using the "cfit" functionality of the MATLAB software. Fitting of the particle locations was done by omitting the first few data points of the damped oscillation, as Eq. (7) only holds for the oscillatory part of the motion. Hence, the acceleration part of the movement is omitted, which does not affect the found resonance frequency and damping time constant. The parameters that were fitted by the MATLAB software on Eq. (7) were as follows: the amplitude of the oscillatory motion $D$, the resonance frequency $\mathbf{f}_{\mathbf{d}}$, the damping time constant $\tau$, the offset $z_{0}$, the initial phase $\phi_{s}$, and an offset in time $t$. Statistical information of the total data set has been obtained by using the "mean" and the "std" basic functions provided in MATLAB.

\section{PROOF-OF-PRINCIPLE EXPERIMENTS}

The feasibility of the proposed method to determine the resonance frequency and damping ratio of a vertically excited microparticle confined in the sheath of a low pressure plasma operated above a horizontal surface is demonstrated in Sec. IV A. The accuracy of this method as a function of the number of experimental repetitions (with exactly the same particle) is discussed in Sec. IV B. Finally, the results are compared with the established resonance method technique in Sec. IV C.

\section{A. Proof-of-concept}

Exemplary experiments of one single probing microparticle subjected to a rising step excitation (i.e., an instantaneous increase in plasma power from $1.5 \pm 0.2$ to $3.2 \pm 0.3 \mathrm{~W}$ ) and a falling step excitation (i.e., an instantaneous decrease in plasma power from $6.6 \pm 0.7$ $\mathrm{W}$ to $3.2 \pm 0.3 \mathrm{~W}$ ) were conducted. The recorded vertical particle position data as a function of time after these excitations are shown in Fig. 4.

As can be seen from this figure, while having different initial $(t<0)$ equilibrium positions, $z_{1}$ is on average $3.519 \pm 0.003 \mathrm{~mm}$ and $z_{\mathrm{h}}$ is on average $3.654 \pm 0.018 \mathrm{~mm}$ for the respective plasma powers of $1.5 \pm 0.2 \mathrm{~W}$ and $6.6 \pm 0.7 \mathrm{~W}$. Nevertheless, the particle is found to oscillate eventually toward the same (on average) equilibrium position $z_{0}=3.555 \mathrm{~mm}$ (the lack of error estimation is explained in Sec. V C) for both the rising and the falling step excitation experiments. Also included in Fig. 4 are fits of Eq. (7) through the experimental data points. These fits clearly demonstrate that-already from a single step excitation - the resonance frequency and the damping ratio of the system can immediately be obtained.

Zooming in on the raw data plot of the falling step excitation experiment shows that there is a missing data point around $t \approx 0.015$. This is caused by the lack of optical signal from the particle due to a too high velocity of the probing microparticle in the captured frames (discussed further in Sec. V B). Hence, such data points are not taken into account when fitting the raw data with Eq. (7). 


\section{B. Determination of the microparticle's resonance frequency and damping ratio}

To investigate the validity and the accuracy of the proposed experimental method and the influence of experiment repetition on it, both the rising step excitation and the falling step excitation experiments presented in Fig. 4 have been repeated 365 times. The raw data of each individual step response has been fitted with Eq. (7), and from the fitting parameters, the decay time constant $\tau$ and the damped resonance frequency $\mathbf{f}_{\mathbf{d}}$ have been obtained. Statistics have been applied to the obtained parameters, from which the standard deviations were estimated. Figures 5 and 6 show, for the rising and for the falling step excitation experiments, the mean values of these parameters together with the standard error on these "means" as a function of the amount of experiment repetitions. Although structural drifts in process parameters (e.g., thermal drifts, see Sec. V C) were insignificant, the experiments making up the subsets used to construct Figs. 5 and 6 have been randomly chosen from the overall set of experiments, of course, while distinguishing between rising and falling step excitation.

The two graphs immediately show the flexibility that this method provides for the experimenter. If measurements need to be done quickly-for instance, in cases where plasma etching of the used microparticles is significant-a few measurements will give a rough estimate of the resonance frequency and damping time constant already. If the experimental situation allows to perform more successive measurements, the accuracy of the overall experiment increases.

From the full sets of experiments (i.e., 365 repetitions for each type of experiment), the values of the means and standard deviations converge to values that are shown in Table I.

From the resulting parameters in this table, it can be concluded that-although relatively close-both the damped resonance frequency $\mathbf{f}_{\mathbf{d}}$ and the corresponding standard deviation $\boldsymbol{\sigma}_{\mathbf{d}}$ for the rising step excitation experiment are slightly higher than those for the falling step excitation experiment. The same trend was found for the values of the decay time $\tau$ and its standard deviation $\boldsymbol{\sigma}_{\tau}$, where it should be noted that the spread in the obtained time constant for the rising step

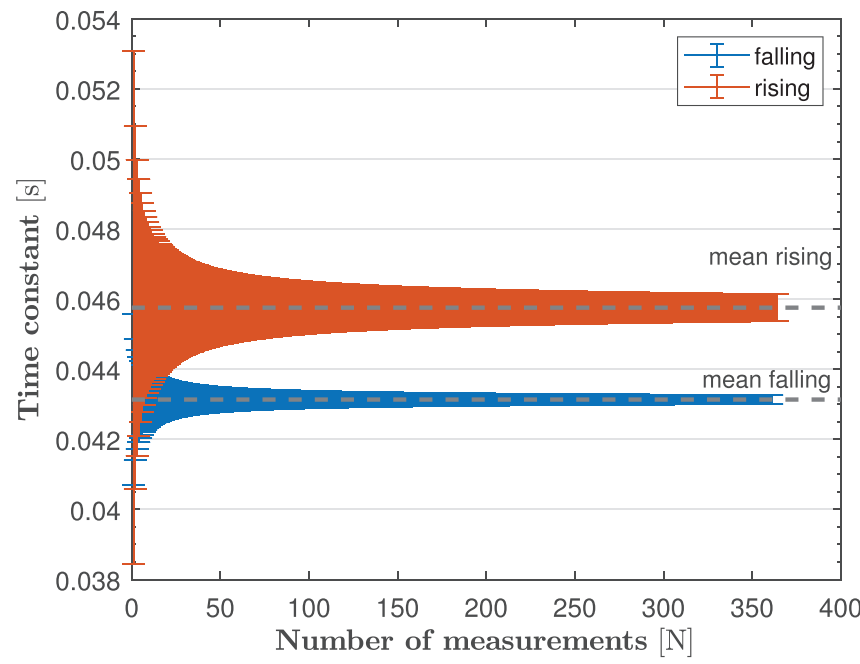

FIG. 5. Standard error on the mean of the fitted decay time constant as a function of the number of repetitions.

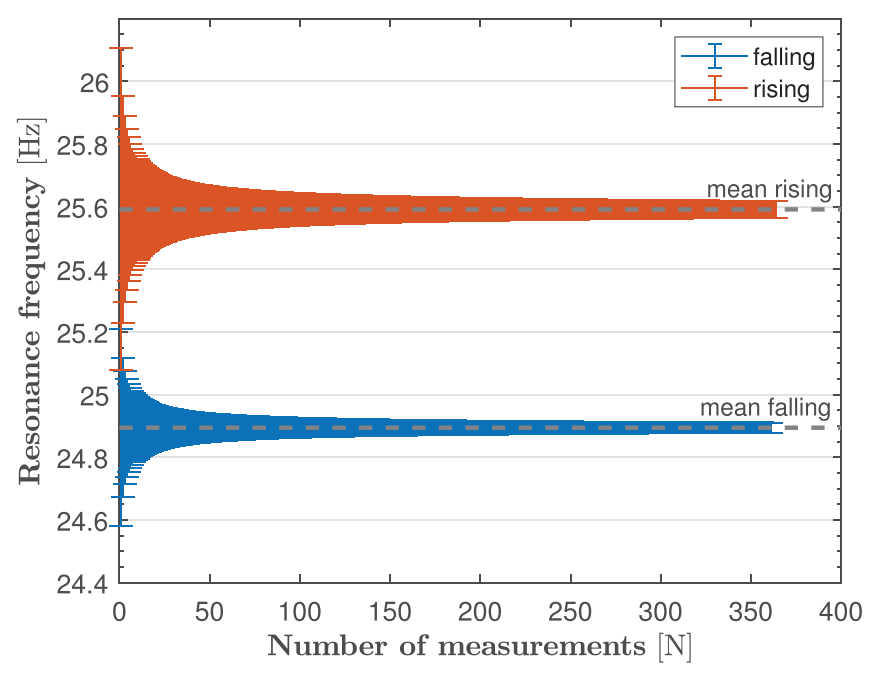

FIG. 6. Standard error on the mean of the fitted resonance frequency as a function of the number of repetitions.

excitation experiment is significantly larger than the spread in the obtained time constant for the falling step excitation experiment. This larger spread is most likely caused by the relatively smaller initial amplitude of the damped oscillation for rising step excitation experiment.

The values of the obtained resonance frequency and the damping ratio together with their standard deviations are used to calculate the natural frequency $\mathbf{f}_{\mathbf{0}}$ by substitution in Eq. (11) (see Table I). The damping ratio $\zeta$ displayed in the same table is calculated from rewriting Eq. (10) and the substitution of the natural frequency and the decay time.

It can be seen from the obtained mean $\zeta$ that the damping ratio of the falling step excitation experiment is $5 \%$ larger compared to the rising step excitation experiment. However, this difference is relatively small when comparing the found damping ratios with the calculated uncertainties.

\section{Comparison with the linear sweep method}

For benchmarking and comparison, a frequency sweep experiment, as done in the work of Melzer et al., ${ }^{35}$ has been conducted with exactly the same microparticle and under identical plasma conditions during one and the same experimental run. To this end, the first function generator (elaborated on in Sec. III A) was programmed to generate a 0.01 to $60.01 \mathrm{~Hz}$ linear increasing frequency sweep at a rate of $0.25 \mathrm{~Hz} \mathrm{~s}^{-1}$ during a total experiment duration of $240 \mathrm{~s}$. (Note that one single step excitation experiment can be conducted on sub-second time scales.) The second function generator modulated-with the

TABLE I. Fitted and calculated parameters for the step-wise excitation experiments.

\begin{tabular}{lcccccc}
\hline \hline & $\mathbf{f}_{\mathbf{d}}(\mathrm{Hz})$ & $\boldsymbol{\sigma}_{\mathbf{d}}(\mathrm{Hz})$ & $\tau(\mathrm{ms})$ & $\boldsymbol{\sigma}_{\tau}(\mathrm{ms})$ & $\mathbf{f}_{\mathbf{0}}(\mathrm{Hz})$ & $\zeta$ \\
\hline Rising & 25.6 & 0.51 & 45.7 & 7.32 & $25.9 \pm 0.6$ & $0.140 \pm 0.024$ \\
Falling & 24.9 & 0.31 & 43.1 & 2.44 & $25.2 \pm 0.3$ & $0.147 \pm 0.010$ \\
\hline
\end{tabular}




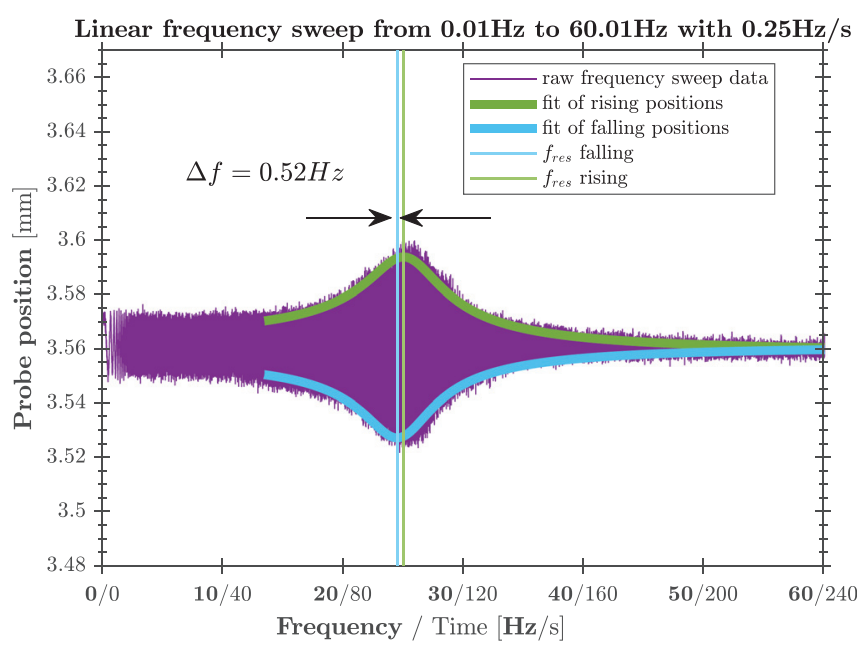

FIG. 7. Raw data of the linear frequency sweep, with two fits through the particle's maxima and minima positions. A discrepancy of $0.52 \mathrm{~Hz}$ is found between the fitted resonance frequencies.

momentary frequency provided by the first function generator-the RF power by $\pm 12 \%$. The microparticle position evolution is plotted in Fig. 7, which was tracked with the high temporal-resolution of 120 FPS during this frequency sweep. The resonant behavior of the microparticle is already clearly visible in the raw data. Table II shows the obtained resonance frequencies and damping ratio from the fits through the particle maxima and minima, i.e., $\mathbf{f}_{\text {res }}$ and $\zeta_{\text {res }}$. The calculated natural frequencies of the probing microparticle are also shown.

First, it can be concluded that the mean of the vertical microparticle equilibrium position, i.e., $z_{0, \text { sweep }}=3.56 \mathrm{~mm}$, perfectly agrees with the average of $z_{0}=3.555 \mathrm{~mm}$ for both step excitation experiments within experimental error bounds (see Fig. 8 for details). This means that the proposed step-wise excitation approach is able to probe the plasma at exactly the same vertical microparticle equilibrium position as is done by traditional resonance techniques.

Second, when comparing the resonance frequency obtained from the frequency sweep measurements with those obtained by the stepwise excitation approach, the following is observed. For the frequency sweep case, we simply determine the resonance frequency $\mathbf{f}_{\text {res }}$ by using resonance curve fits in the time domain through the maxima and minima in the microparticle positions. As can be seen from Fig. 7, the fit through the maxima delivers a $\mathbf{f}_{\text {res }}=25.1 \mathrm{~Hz}$, which is somewhat higher than the $\mathbf{f}_{\text {res }}=24.5 \mathrm{~Hz}$ obtained from the fit through the minima. Notably, this is in the same direction as the discrepancy observed between the damped resonance frequencies for the rising step excitation $\left(\mathbf{f}_{\mathbf{d}}=25.6 \mathrm{~Hz}\right)$ and the falling step excitation $\left(\mathbf{f}_{\mathbf{d}}=24.9 \mathrm{~Hz}\right)$ and

TABLE II. Fitted parameters of the top and bottom particle locations of the linear frequency sweep.

\begin{tabular}{lccc}
\hline \hline Frequency sweep $(\mathrm{Hz} / \mathrm{s})$ & $\mathbf{f}_{\text {res }}(\mathrm{Hz})$ & $\boldsymbol{\zeta}_{\text {res }}$ & $\mathbf{f}_{\mathbf{0}}(\mathrm{Hz})$ \\
\hline Maxima & 25.05 & 0.122 & 25.43 \\
Minima & 24.53 & 0.115 & 24.85 \\
\hline \hline
\end{tabular}

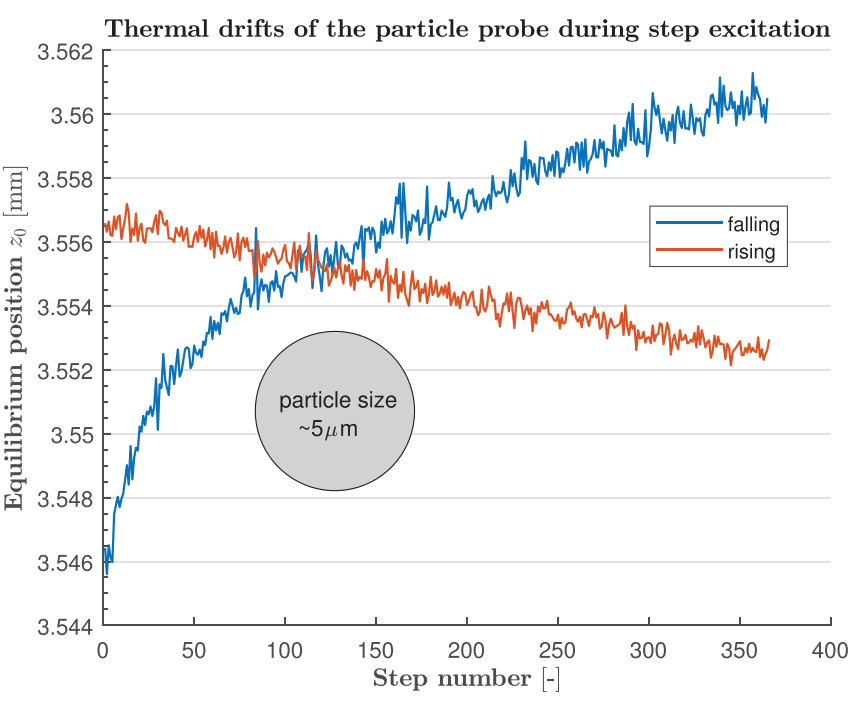

FIG. 8. Fitted equilibrium position $z_{0}$ of both the rising and falling step excitation experiments, together with the particle size on the same scale.

may possibly originate from a slightly non-linear sheath structure. Overall, regardless of the direction of step excitation (rising or falling), it can be concluded that the obtained damped resonance frequencies are within $5 \%$ with respect to those values obtained from the traditional resonance method.

Since the damping ratios for both methods, i.e., step excitation and frequency sweep, are close together as well, it is clear that also the obtained natural resonance frequencies $\mathbf{f}_{\mathbf{0}}$ from both methods match within $5 \%$ accuracy.

\section{ADDITIONAL DISCUSSION}

In addition to the discussion of the proof-of-principle experiments and the comparison of those with the linear frequency sweep method, this section elaborates on three important topics as follows: comparing the experimentally found damping rates with the classical damping rate predicted by Epstein, optimally resolving the particle trajectories, and the (thermal) drift of the microparticle's probing location during the total measurement time.

\section{A. Comparison of the damping rates with the theory}

In this work, the obtained damping ratios can be compared to the classical damping rate defined by Epstein et al. The Epstein damping rate is defined as ${ }^{52} \beta_{\text {eps }}=\delta \frac{4}{\pi} \frac{p}{\rho v_{\text {th }} r_{\mathrm{p}}}$, where $p$ is the gas pressure, $v_{\text {th }}$ is the thermal velocity of the neutrals, and $\delta$ is a dimensionless factor that depends on whether the neutrals collide with the particle's surface by diffusive or by specular reflections (1.44 or 1, respectively) of. ${ }^{53}$ When calculating the Epstein damping rate for the parameters used in this work, one obtains $\beta_{\text {eps }}=16.2 \pm 1.7 \mathrm{~s}^{-1}$ assuming diffuse reflections. Converting the experimentally found $\zeta$ to the Epstein damping rate via $\zeta * \omega_{0}=\beta$, for the rising and falling step excitation experiments, the values of $22.8 \pm 3.9$ and $23.3 \pm 1.6 \mathrm{~s}^{-1}$ are found, respectively. Both values are larger than the damping rate predicted by Epstein et al. Calculating the same damping rates for the results of the linear frequency sweep, the maxima and minima of the results in 
Table II give damping rates of 19.49 and $17.96 \mathrm{~s}^{-1}$, respectively. Hence, it is clear that the experimentally found values for the damping rate deviate more in the case of the step excitation method (compared to the frequency sweep method). This might be caused by the relatively large steps in plasma power, used to excite the probing particle to a sufficient extent. Pustylnik et al. have reported that the classic Epstein damping rate should be corrected by the so-called Delayed Charging Effect, ${ }^{52}$ which is dependent on the plasma parameters and is defined as $\beta_{\mathrm{DCE}}=\left.\frac{\tau_{\mathrm{ch}}}{2} \frac{e E}{m_{\mathrm{p}}} \frac{\partial Q_{\mathrm{p}}}{\partial z}\right|_{z=z_{0}}$, where $\tau_{\mathrm{ch}}$ is the charging time. According to Pustylnik et al., in such cases the damping rates can be summed to a total damping rate as $\beta=\beta_{\text {eps }}+\beta_{\mathrm{DCE}}$. In conclusion, this means that the step excitation method introduced here is more influenced by the Delayed Charging Effect than the linear frequency sweep method is.

\section{B. Resolving the particle trajectories}

For optimally resolving the microparticle's damped oscillatory trajectory, there is always a trade-off between, on one side, a sufficiently large oscillation amplitude in order to achieve sufficient spatial resolution and, on the other side, a sufficiently low velocity of the microparticle to have sufficient optical signal to detect the particle at all times.

In the view of achieving sufficiently large oscillation amplitudes, the applied step excitation method needs-in general-more power modulation $(\approx 50 \%$ and $\approx 100 \%$ for the rising and falling probe experiments, respectively) than the linear frequency sweep method requires (in the case of this work $\approx 12 \%$ ). However, as discussed before, due to the much shorter plasma dynamics time scales compared to the microparticle dynamics time scales, these time scales can be considered fully decoupled in the case of step excitation, while this may not be the case for the linear frequency sweep method.

Furthermore, it is not surprising that the standard deviations on the mean values of $\mathbf{f}_{\mathbf{d}}$ and $\tau$ for the rising step excitation experiments (having a relatively small initial oscillation amplitude) are significantly higher than those for the falling step excitation experiments (having a relatively large initial oscillation amplitude). At the same time, just after the step excitation in the falling probe case, the microparticle's velocity is too high such that the optical system and post-processing fail to detect the first few data points, as can be seen in Fig. 4.

Overall, as the optical irradiation and detection systems seem to be the limiting factor in resolving the probing microparticle, a more powerful/focused laser beam enables faster and less noisy camera images. Here, the experimenter must be aware of the risk of additional and unwanted effects, such as higher radiation pressure, increased particle heating, light scattering by the vacuum vessel etcetera. Additionally, a more sensitive/intensified camera can be used to enhance the measurements' signal-to-noise ratio.

\section{C. (Thermal) drift of the microparticle's probing location}

With the step excitation with a $50 \%$ duty cycle, the time averaged plasma power for the rising step excitation (from $1.5 \pm 0.2 \mathrm{~W}$ to $3.2 \pm 0.3 \mathrm{~W}$ ) is $2.4 \mathrm{~W}$. This is much lower than the plasma power used in the falling step excitation experiments (from $6.6 \pm 0.7$ to $3.2 \pm 0.3 \mathrm{~W}$ ), being $4.9 \mathrm{~W}$ on average. Assuming that a significant fraction of this power is deposited on the bottom (RF powered) electrode of this asymmetric discharge, it can be assumed that the thermal load on the bottom electrode during the falling step excitation experiments is higher than that during the rising step excitation experiments. To investigate the possible disturbing effect of an additional upward thermophoretic force on the overall equilibrium position of the particle, the microparticle's equilibrium position $\left(z_{0}\right)$ as a function of consecutive step excitation experiments is plotted in Fig. 8 for both the rising and falling step excitation experiments.

As can be seen from this figure, a drift in particle equilibrium position of the microparticle is indeed observed. This drift is-as described above-upwards for consecutive falling step excitation experiments and downwards for consecutive rising step excitation experiments. However, this drift is astonishingly small with a maximum drift in either direction of only $14 \mu \mathrm{m}$ for a measurement duration of $183 \mathrm{~s}$. For comparison, the size of the used microparticle is plotted in the figure as well, highlighting not only the high spatial resolution of the used optical system and post-processing, but more importantly also the stability of the microparticle's probing position.

This effect can be fully mitigated by allowing the setup to reach thermal equilibrium with the same average plasma power used during the step excitation waveform. For example, if the experimenter wants to perform the rising step excitation experiment with a duty cycle of the excitation wave of $50 \%{ }^{\circ}$ and power steps from 1.5 to $3.2 \mathrm{~W}$, the constant (no modulation) plasma setting for reaching the thermal equilibrium should be set at $2.35 \mathrm{~W}$. Of course, the same approach works for reaching the required thermal equilibrium with respect to the falling step excitation experiment.

\section{CONCLUSION}

We have introduced a new step-wise excitation approach to quickly obtain, or with high accuracy, the resonance frequency of a microparticle confined in the space charge region of a low pressure plasma operated above a horizontal surface. This step-wise excitation was achieved by suddenly changing the plasma power, which instantaneously altered the equilibrium position of the confined microparticle as well. By resolving the trajectory of the microparticle toward its new equilibrium position and comparing this trajectory with the theory describing the damped harmonic oscillator, the local value of the resonance frequency can be retrieved.

Such a resonance frequency, together with the obtained damping ratio, can be used to calculate the natural frequency of the particle probe. This, in turn, is essential to obtain for the interpretation of many dusty and complex plasma laboratory experiments, because it can directly be related to the particle's charge.

The step-wise excitation approach was compared to the traditional frequency sweep particle resonance method and the results of both methods match one another within $5 \%$.

The found damping rates are compared to the classical Epstein damping rate, where the results from the step excitation experiments show a larger discrepancy from the classic damping rate, than the results from the linear frequency sweep do. This is most likely caused by the Delayed Charging Effect.

With the proof-of-principle experiments presented in this work, we have demonstrated the flexibility that this method provides for the experimenter. If measurements need to be done quickly-for instance, in cases where plasma etching of the used microparticles is significant-a few measurements (with sub-second measurement time per experiment repetition) will give a rough estimate of the resonance 
frequency and the damping time constant. If the experimental situation allows time to perform more successive measurements, the accuracy of the parameter determination increases.

\section{ACKNOWLEDGMENTS}

The authors would like to thank A. B. Schrader and P. Sanders for their skillful technical support.

\section{DATA AVAILABILITY}

The data that support the findings of this study are available from the corresponding author upon reasonable request.

\section{REFERENCES}

${ }^{1}$ G. S. Selwyn, J. Singh, and R. S. Bennett, "In situ laser diagnostic studies of plasmagenerated particulate contamination," J. Vacuum Sci. Technol. A 7, 2758-2765 (1989).

${ }^{2}$ V. E. Fortov, A. V. Ivlev, S. A. Khrapak, A. G. Khrapak, and G. E. Morfill, "Complex (dusty) plasmas: Current status, open issues, perspectives," Phys. Rep. 421, 1-103 (2005).

${ }^{3}$ G. E. Morfill and A. V. Ivlev, "Complex plasmas: An interdisciplinary research field,” Rev. Mod. Phys. 81, 1353-1404 (2009).

${ }^{4}$ K.-S. Kim and M. Ikegawa, "Particle growth and transport in silane plasma chemical vapour deposition," Plasma Sources Sci. Technol. 5, 311-322 (1996).

${ }^{5}$ W. Stoffels, E. Stoffels, G. Ceccone, and F. Rossi, "Formation of particle conglomerates in a methane discharge," in Frontiers in Dusty Plasmas, edited by Y. Nakamura, T. Yokota, and P. K. Shukla (Elsevier Science B.V., 2000), pp. 493-496.

${ }^{6}$ S. Groth, F. Greiner, and A. Piel, "Spatio-temporally resolved investigations of layered particle growth in a reactive argon-acetylene plasma," Plasma Sources Sci. Technol. 28, 115016 (2019).

${ }^{7}$ Z. Marvi, E. V. Wahl, T. Trottenberg, and H. Kersten, "Spatiotemporal sampling of growing nanoparticles in an acetylene plasma," J. Appl. Phys. 127, 173301 (2020).

${ }^{8}$ H. Kakiuchi, H. Ohmi, T. Yamada, K. Yokoyama, K. Okamura, and K. Yasutake, "Silicon oxide coatings with very high rates $(>10 \mathrm{~nm} / \mathrm{s})$ by hexamethyldisiloxane-oxygen fed atmospheric-pressure VHF plasma: Filmforming behavior using cylindrical rotary electrode," Plasma Chem. Plasma Process. 32, 533-545 (2012).

${ }^{9} \mathrm{P}$. Agarwal and S. L. Girshick, "Numerical modeling of an RF argon-silane plasma with dust particle nucleation and growth," Plasma Chem. Plasma Process. 34, 489-503 (2014).

${ }^{10}$ H. Okuno, E. Grivei, F. Fabry, T. M. Gruenberger, J. Gonzalez-Aguilar, A. Palnichenko, L. Fulcheri, N. Probst, and J.-C. Charlier, "Synthesis of carbon nanotubes and nano-necklaces by thermal plasma process," Carbon 42, 2543-2549 (2004).

${ }^{11}$ A. A. Howling, L. Sansonnens, J. Dorier, and C. Hollenstein, "Timeresolved measurements of highly polymerized negative ions in radio frequency silane plasma deposition experiments," J. Appl. Phys. 75, 1340-1353 (1994).

${ }^{12}$ J. H. Chu and L. I, "Direct observation of Coulomb crystals and liquids in strongly coupled rf dusty plasmas,” Phys. Rev. Lett. 72, 4009-4012 (1994).

${ }^{13}$ H. Thomas, G. E. Morfill, V. Demmel, J. Goree, B. Feuerbacher, and D. Möhlmann, "Plasma crystal: Coulomb crystallization in a dusty plasma," Phys. Rev. Lett. 73, 652-655 (1994).

${ }^{14}$ V. A. Schweigert, I. V. Schweigert, A. Melzer, A. Homann, and A. Piel, "Plasma crystal melting: A nonequilibrium phase transition," Phys. Rev. Lett. 80, 5345-5348 (1998).

${ }^{15} \mathrm{~J}$. B. Pieper and J. Goree, "Dispersion of plasma dust acoustic waves in the strong-coupling regime,” Phys. Rev. Lett. 77, 3137-3140 (1996).

${ }^{16}$ S. Khrapak, D. Samsonov, G. Morfill, H. Thomas, V. Yaroshenko, H. Rothermel, T. Hagl, V. Fortov, A. Nefedov, V. Molotkov, O. Petrov, A. Lipaev, A. Ivanov, and Y. Baturin, "Compressional waves in complex (dusty) plasmas under microgravity conditions," Phys. Plasmas 10, 1-4 (2003).
${ }^{17}$ Y.-M. Sung and H.-J. Kim, "Sputter deposition and surface treatment of TiO2 films for dye-sensitized solar cells using reactive RF plasma," Thin Solid Films 515, 4996-4999 (2007).

${ }^{18}$ M. A. Hartney, D. W. Hess, and D. S. Soane, "Oxygen plasma etching for resist stripping and multilayer lithography,” J. Vacuum Sci. Technol., B 7, 1 (1989).

${ }^{19} \mathrm{M}$. A. Lieberman and A. J. Lichtenberg, Priciples of Plasma Discharges and Materials Processing, 2nd ed. (John Wiley \& Sons, Inc., 2005).

${ }^{20}$ L. Yuan, C. Chen, S. Shyu, and J. Lai, "Plasma surface treatment on carbon fibers. Part 1: Morphology and surface analysis of plasma etched fibers," Compos. Sci. Technol. 45, 1-7 (1992).

${ }^{21}$ B. van Minderhout, J. C. van Huijstee, B. Platier, T. Peijnenburg, P. Blom, G. M. Kroesen, and J. Beckers, "Charge control of micro-particles in a shielded plasma afterglow,” Plasma Sources Sci. Technol. 29, 065005 (2020).

${ }^{22}$ H. M. Mott-Smith and I. Langmuir, "The theory of collectors in gaseous discharges," Phys. Rev. 28, 727-763 (1926).

${ }^{23}$ W. L. Wiese, D. E. Kelleher, and D. R. Paquette, "Detailed study of the stark broadening of balmer lines in a high-density plasma," Phys. Rev. A 6, 1132-1153 (1972).

${ }^{24}$ W. B. Nottingham, R. H. Good, E. W. Müller, R. Kollath, G. L. Weissler, W. P. Allis, L. B. Loeb, A. von Engel, and P. F. Little, Electron-Emission Gas Discharges I/Elektronen-Emission Gasentladungen I, 1st ed. Encyclopedia of Physics/Handbuch Der Physik, Vol. 4/21 (Springer, Berlin/Heidelberg, 1956).

${ }^{25}$ A. A. Samarian and B. W. James, "Dust as fine electrostatic probes for plasma diagnostic," Plasma Phys. Controlled Fusion 47, B629-B639 (2005).

${ }^{26}$ V. Land, B. Smith, L. Matthews, and T. Hyde, "Probing the sheath electric field with a crystal lattice by using thermophoresis in dusty plasma," IEEE Trans. Plasma Sci. 38, 768-773 (2010).

${ }^{27}$ P. Hartmann, A. Z. Kovács, J. C. Reyes, L. S. Matthews, and T. W. Hyde, "Dust as probe for horizontal field distribution in low pressure gas discharges," Plasma Sources Sci. Technol. 23, 045008 (2014).

${ }^{28}$ A. Douglass, V. Land, K. Qiao, L. Matthews, and T. Hyde, "Using dust as probes to determine sheath extent and structure," J. Plasma Phys. 82, 615820402 (2016).

${ }^{29}$ L. Schepers, J. Beckers, and W. IJzerman, "Determination of microparticle characteristics in an etching plasma," Contrib. Plasma Phys. 58, 985-994 (2018).

${ }^{30}$ K. S. Ashrafi, R. Yousefi, M. Chen, L. S. Matthews, and T. W. Hyde, "Dust as probes: Determining confinement and interaction forces,” Phys. Rev. E 102, 043210 (2020).

${ }^{31}$ J. Kong, K. Qiao, L. S. Matthews, and T. W. Hyde, "Dust particle pair correlation functions and the nonlinear effect of interaction potentials," IEEE Trans. Plasma Sci. 47, 3057-3062 (2019).

${ }^{32} \mathrm{~V}$. Schneider and H. Kersten, "An optical trapping system for particle probes in plasma diagnostics,” Rev. Sci. Instrum. 89, 103505 (2018).

${ }^{33}$ E. B. Tomme, B. M. Annaratone, and J. E. Allen, "Damped dust oscillations as a plasma sheath diagnostic," Plasma Sources Sci. Technol. 9, 87-96 (2000).

${ }^{34}$ V. Schneider, T. Trottenberg, I. Teliban, and H. Kersten, "An experiment for the investigation of forces on microparticles in ion beams," Rev. Sci. Instrum. 81, 013503 (2010).

${ }^{35}$ A. Melzer, T. Trottenberg, and A. Piel, "Experimental determination of the charge on dust particles forming Coulomb lattices," Phys. Lett. A 191, 301-308 (1994).

${ }^{36}$ A. Homann, A. Melzer, and A. Piel, "Measuring the charge on single particles by laser-excited resonances in plasma crystals," Phys. Rev. E 59, R3835-R3838 (1999).

${ }^{37}$ C. Zafiu, A. Melzer, and A. Piel, "Nonlinear resonances of particles in a dusty plasma sheath,” Phys. Rev. E 63, 066403 (2001).

${ }^{38}$ J. Carstensen, H. Jung, F. Greiner, and A. Piel, "Mass changes of microparticles in a plasma observed by a phase-resolved resonance method," Phys. Plasmas 18, 033701 (2011).

${ }^{39}$ W. W. Stoffels, E. Stoffels, G. H. P. M. Swinkels, M. Boufnichel, and G. M. W. Kroesen, "Etching a single micrometer-size particle in a plasma," Phys. Rev. E 59, 2302-2304 (1999).

${ }^{40}$ N. Kohlmann, F. Wieben, O. H. Asnaz, D. Block, and F. Greiner, "High-precision in-situ size measurements of single microparticles in an RF plasma," Phys. Plasmas 26, 053701 (2019). 
${ }^{41}$ Dusty Plasmas: Physics, Chemistry, and Technological Impacts in Plasma Processing, edited by A. Bouchoule (John Wiley \& Sons, Inc., 1999).

${ }^{42}$ J. Beckers, T. Ockenga, M. Wolter, W. W. Stoffels, J. van Dijk, H. Kersten, and G. M. W. Kroesen, "Microparticles in a collisional Rf plasma sheath under hypergravity conditions as probes for the electric field strength and the particle charge," Phys. Rev. Lett. 106, 115002 (2011).

${ }^{43}$ S. A. Khrapak, S. V. Ratynskaia, A. V. Zobnin, A. D. Usachev, V. V. Yaroshenko, M. H. Thoma, M. Kretschmer, H. Höfner, G. E. Morfill, O. F. Petrov, and V. E. Fortov, "Particle charge in the bulk of gas discharges," Phys. Rev. E 72, 016406 (2005).

${ }^{44}$ S. A. Khrapak, A. V. Ivlev, G. E. Morfill, and H. M. Thomas, "Ion drag force in complex plasmas," Phys. Rev. E 66, 046414 (2002).

${ }^{45}$ M. S. Barnes, J. H. Keller, J. C. Forster, J. A. O’Neill, and D. K. Coultas, "Transport of dust particles in glow-discharge plasmas," Phys. Rev. Lett. 68, 313-316 (1992).

${ }^{46}$ J. A. Bittencourt, Fundamentals of Plasma Physics: Introduction, 3rd ed. (Springer-Verlag, New York, 2010).

${ }^{47}$ X.-F. Wang, W.-Z. Jia, Y.-H. Song, Y.-Y. Zhang, Z.-L. Dai, and Y.-N. Wang, "Hybrid simulation of electron energy distributions and plasma characteristics in pulsed RF CCP sustained in $\mathrm{Ar}$ and $\mathrm{SiH}_{4} / \mathrm{Ar}$ discharges," Phys. Plasmas 24, 113503 (2017).

${ }^{48}$ A. Omran and B. Newman, "Analytical response for the prototypic nonlinear mass-spring-damper system," in ASME 10th Biennial Conference on Engineering Systems Design and Analysis (ASMEDC, Istanbul, Turkey, 2010), Vol. 5, pp. 43-52.

${ }^{49} \mathrm{C}$. Schmidt and A. Piel, "Stochastic heating of a single Brownian particle by charge fluctuations in a radio-frequency produced plasma sheath," Phys. Rev. E 92, 043106 (2015).

${ }^{50}$ L. C. J. Heijmans, "Quantifying plasma particle lofting," Ph.D. thesis (Eindhoven University of Technology, 2017).

${ }^{51}$ B. van Minderhout, T. Peijnenburg, P. Blom, J. M. Vogels, G. M. W. Kroesen, and J. Beckers, "The charge of micro-particles in a low pressure spatial plasma afterglow,” J. Phys. D 52, 32LT03 (2019).

${ }^{52}$ M. Y. Pustylnik, N. Ohno, S. Takamura, and R. Smirnov, "Modification of the damping rate of the oscillations of a dust particle levitating in a plasma due to the delayed charging effect," Phys. Rev. E 74, 046402 (2006).

${ }^{53} \mathrm{P}$. S. Epstein, "On the resistance experienced by spheres in their motion through gases," Phys. Rev. 23, 710-733 (1924). 